\title{
THE STABILITY OF VORTICES IN GAS ON THE l-PLANE: THE INFLUENCE OF CENTRIFUGAL FORCE
}

\author{
OLGA ROZANOVA, MARKO TURZYNSKY
}

\begin{abstract}
We show that a small correction due to centrifugal force usually neglected in the $l$-plane model of atmosphere drastically influences on the stability of vortices. Namely, in the presence of the Coriolis force only there exists a wide range of parameter ensuring nonlinear stability of a vortex with uniform deformation. Taking into account the centrifugal force results in a disappearance of stable vortices in the above mentioned class of motions.

We also prove that for the heat ratio $\gamma=2$, corresponding to the one-atomic gas, the system of equations, describing the gas on the $l$ plane with the correction due to centrifugal force can be integrated in a special case.
\end{abstract}

\section{Reduction of 3D primitive EQuations of the ATMosphere DYNAMICS TO THE $l$-PLANE MODEL}

We consider the system of gas dynamics, widely used for a description of the dynamics of atmosphere of a rotating planet, such as the Earth. We do not dwell on the processes of heat and moisture transfer and study the behavior of atmosphere in the frame of the model of ideal polytropic inviscid gas. The model consists of the following equations [15]

$$
\begin{gathered}
\rho\left(\partial_{t} \bar{U}+(\bar{U}, \nabla) \bar{U}+2 \bar{\omega} \times \bar{U}+\bar{\omega} \times(\bar{\omega} \times \bar{r})+g \bar{e}_{3}\right)=-\nabla p, \\
\partial_{t} \rho+\operatorname{div}(\rho \bar{U})=0, \\
\partial_{t} S+(\bar{U}, \nabla S)=0,
\end{gathered}
$$

for $\rho \geq 0, p \geq 0, \bar{U}=\left(U_{1}, U_{2}, U_{3}\right), S$ (density, pressure, velocity and entropy). The functions depend on time $t$ and on point $x \in \mathbb{R}^{3}, \bar{e}_{3}=(0,0,1)$ is the "upward" unit vector, $g$ is the acceleration due to gravity (points in $-e_{3}$ direction to the center of planet), $\bar{\omega}$ is the velocity of angular rotation, $\bar{r}$ is the radius-vector of point in the coordinate system, associated with the center of planet. The state equation is

$$
p=\rho^{\gamma} e^{S}, \quad \gamma>1 .
$$

System (11) - (3) describes a layer of air over a spherical planet, therefore for the processes of planetary scale one should use spherical coordinates. Nevertheless, for the process of so called middle scale it is possible to fix a

Key words and phrases. compressible 2D medium, vortex motion, stability, rotation, centrifugal force. 
point on the Earth surface and consider a coordinate system with the origin in these point. Thus, axes $x_{1}$ and $x_{2}$ are directed in the tangent plane along parallel and meridian, respectively, and the axis $x_{3}$ is directed out of the center of planet.

Let us compute the term $\bar{\omega} \times(\bar{\omega} \times \bar{r})$ for the point lying on the plane $x_{3}=0$. Thus, the coordinates of the radius-vector $\bar{r}$ are $\left(x_{1}, x_{2}, R\right)$, where $R$ is the radius of planet. If we denote $\theta$ the latitude of the origin of the rotating coordinate system and take into account that $\bar{\omega}=(0, \omega \cos \theta, \omega \sin \theta), \omega=$ $|\bar{\omega}|$, then

$$
\bar{\omega} \times(\bar{\omega} \times \bar{r})=R \omega^{2}\left(0, \sin \theta \cos \theta,-\cos ^{2} \theta\right)-\omega^{2}\left(x_{1}, x_{2} \sin ^{2} \theta, x_{2} \sin \theta \cos \theta\right) .
$$

The first vector in this sum is constant, the second one depends on the position of point on the plane $x_{3}=0$.

Since $\bar{\omega} \times(\bar{\omega} \times \bar{r})=-\frac{1}{2} \nabla \omega^{2}|\bar{r}|^{2}$, traditionally they include this term in the geopotential $\Phi=g x_{3}-\frac{1}{2} \omega^{2}|r|^{2}$ and turn the axis $\left(x_{2}, x_{3}\right)$ such that the constant vector

$$
R \omega^{2}\left(0, \sin \theta \cos \theta,-\cos ^{2} \theta\right)-(0,0, g)
$$

in the new coordinate system $\left(x_{1}, \tilde{x}_{2}, \tilde{x}_{3}\right)$ is directed strictly vertically. In fact, we should turn the coordinate system by the angle $\xi$ such that

$$
\cos \xi\left(-R \omega^{2} \sin \theta \cos \theta\right)-\sin \xi\left(-g+R \omega^{2} \cos ^{2} \theta\right)=0,
$$

therefore

$$
\tan \xi=\frac{R \omega^{2} \sin \theta \cos \theta}{g-R \omega^{2} \cos ^{2} \theta} .
$$

We can see that $\xi \ll 1$, since $\frac{R \omega^{2}}{g} \ll 1$.

Thus, we change the spherical surface of planet to the geopotential surface and regard the surfaces of constant $\Phi$ as being true spheres. The horizontal component of apparent gravity is then identically zero (see the details in [15, Sec.2.2.1).

If we are exactly at the origin, the term $\omega^{2}\left(x_{1}, x_{2} \sin ^{2} \theta, x_{2} \sin \theta \cos \theta\right)$ is zero. However, in the general case

$$
k=\cos \xi \sin ^{2} \theta+\sin \xi \sin \theta \cos \theta \neq 0, \quad k \approx \sin ^{2} \theta,
$$

and this means that the centrifugal force still has a projection on the new horizontal coordinates $\left(x_{1}, \tilde{x}_{2}\right)$ as $\left(-\omega^{2} x_{1},-k \omega^{2} \tilde{x}_{2}\right)$. Of course, one can include these terms in the gradient of some new pressure, however this would change the state equation.

Let us repeat the standard procedure of deriving the $l$-plane model for relatively thin atmosphere, ignoring vertical processes (e.g. [15, Sec.2.3.1). They define a plane tangent to the surface of the earth at a fixed latitude $\theta$, and then use a Cartesian coordinate system $\left(x_{1}, x_{2}\right)$ to describe motion on that plane (we will write $x_{2}$ instead of our previous $\tilde{x}_{2}$ ). For small excursions on the plane, they fix the projection of $\bar{\omega}$ in the direction of the local vertical, ignore the components of $\bar{\omega}$ in the horizontal direction, set 
the component of velocity $U_{3}=0$, and make the hydrostatic approximation, which means that the gravitational term is assumed to be balanced by the pressure gradient term. Thus the $3 \mathrm{D}$ vectorial equation (11) results in the following $2 \mathrm{D}$ vectorial equation:

$$
\rho\left(\partial_{t} \mathbf{u}+(\mathbf{u} \cdot \nabla) \mathbf{u}+\mathcal{L} \mathbf{u}-\delta \omega^{2} \mathcal{D} \mathbf{x}\right)+\nabla p=0,
$$

where $\mathbf{u}(t, x)=\left(U_{1}, U_{2}\right), x \in \mathbb{R}^{2}, \mathbf{x}$ is a radius-vector of point, $\mathcal{L}=l L$, $L=\left(\begin{array}{rr}0 & -1 \\ 1 & 0\end{array}\right), l=2 \omega \sin \theta>0$ is the Coriolis parameter, $\mathcal{D}=\left(\begin{array}{ll}1 & 0 \\ 0 & k\end{array}\right)$, $k=\sin ^{2} \theta$. Equations (2), (3) and (4) are not changed formally, however, the density, pressure and entropy depend now only on $x \in \mathbb{R}^{2}$ and $t$. In the isentropic case the equation (3) is valid identically. The value of $\delta$ is zero if we do not consider the centrifugal term or one, otherwise.

The reduction to the $2 \mathrm{D}$ system for a thin layer of atmosphere can be obtained more strictly by averaging over the height, see [7]. The case $\gamma=2$ corresponds to the 2D one-atomic gas $\left(\gamma=2=1+\frac{1}{n}\right.$, where $n$ is the dimension of space). Also it arises in the shallow water equations.

\section{Motion With UNiform DEFORMATION: STEADY STATES AND THEIR STABILITY}

As is well known, the gas dynamics system has a special class of solutions, characterized be a linear profile of velocity, that is

$$
\mathbf{u}(t, \mathbf{x})=Q \mathbf{x}, \quad Q=\left(\begin{array}{cc}
a(t) & b(t) \\
c(t) & d(t)
\end{array}\right) .
$$

In 12 the system (5), (2), (3) was considered for $\delta=0$ and it was shown that the components of matrix $Q$ can be found as a part of solution of a certain quadratically-nonlinear matrix system of equations. For the case of arbitrary $\delta$ this system has the form

$$
\begin{gathered}
\dot{R}+R Q+Q^{T} R+(\gamma-1) \operatorname{tr} Q R=0, \\
\dot{Q}+Q^{2}+l L Q+2 c_{0} R-\delta \omega^{2} \mathcal{D}=0,
\end{gathered}
$$

where the symmetric matrix $R$ describes distribution of density and pressure, $c_{0}=$ const.

2.1. $\delta=0$. System (7), (9) has an one-parametric family of nontrivial equilibria, depending on the parameter $b_{*}$ :

$$
a=d=0, b=-c=b_{*}, A=C=\frac{b_{*}\left(b_{*}-l\right)}{2 c_{0}}, B=0,
$$

and a two-parametric family with parameters $a_{*}$ and $c_{*}$

$$
a=a_{*}, c=c_{*}, b=-\frac{a_{*}^{2}}{c_{*}}, d=-a_{*}, A=\frac{l c_{*}}{2 c_{0}}, B=-\frac{l a_{*}}{c_{0}}, C=\frac{l a_{*}^{2}}{2 c_{0} c_{*}},
$$


for $c_{*}=0$ the latter equilibrium should be completed by

$$
a=c=d=0, b=b_{*}, A=B=0, C=-\frac{1}{2 c_{0}} l b_{*},
$$

with a parameter $b_{*}$.

Equilibrium (9) corresponds to the case of axisymmetric vortex. For $b_{*} \in(0, l)$ the motion is anticyclonic with a higher pressure in the center of vortex (anticyclonic high), for $b_{*}<0$ the motion is cyclonic with a lower pressure in the center of vortex (cyclonic low), and at last, for $b_{*}>l$ the motion is anticyclonic with a higher pressure in the center (anticyclonic low).

Equilibria (10), (11) correspond to a shear flow along a straight line, the ratio $\frac{a_{*}}{c_{*}}$ gives the inclination of this line with respect to the coordinate axis.

Let us give a review of the results about stability of the equilibria.

Theorem 2.1. Equilibrium (9) is unstable for any $\gamma>1$ if

$$
\frac{b_{*}}{l} \in \mathbb{R} \backslash \Sigma \text {, where } \Sigma=\left[K_{-}, K_{+}\right], K_{ \pm}=\frac{1 \pm \sqrt{2}}{2} .
$$

Moreover, for $\gamma>4$ the domain $\Sigma$ can be replaced by $\sigma=\left[k_{-}, k_{+}\right], k_{ \pm}=$ $\frac{1 \pm \sqrt{\gamma /(\gamma-2)}}{2}$. Thus, $\sigma=\left[k_{-}, k_{+}\right] \rightarrow[0,1]$ as $\gamma \rightarrow \infty$ and the domain of instability enlarges.

Proof. Eigenvalues of matrix of linearization in the point (9) for the system (7), (8), written as 7 equations are the following:

$$
\begin{gathered}
\lambda_{1,2,3,4}= \pm \sqrt{2} \sqrt{-l\left(b_{*}+\frac{l}{4}\right) \pm \sqrt{\left(b_{*}+\frac{l}{2}\right)^{2}\left(\frac{l^{2}}{4}+b_{*} l-\left(b_{*}\right)^{2}\right)}}, \\
\lambda_{5,6}= \pm \sqrt{-\left(2(2-\gamma) b_{*}\left(b_{*}-l\right)+l^{2}\right)}, \quad \lambda_{7}=0 .
\end{gathered}
$$

For $b_{*}<\frac{1-\sqrt{2}}{2} l$ and $b_{*}>\frac{1+\sqrt{2}}{2} l>l$ we get $\lambda_{3,4,5,6}= \pm \alpha \pm i \beta, \alpha \neq 0, \beta \neq$ 0 , therefore there exists an eigenvalue with a positive real part. Thus, the Lyapunov theorem implies instability of the equilibrium.

For $\gamma \in(1,2]$ (this case was considered in [12] ) the value $-\left(2(2-\gamma) b_{*}\left(b_{*}-\right.\right.$ $\left.l)+l^{2}\right)<0$, then $\Re\left(\lambda_{1,2}\right)=0$. For $\gamma>2$ we have $-\left(2(2-\gamma) b_{*}\left(b_{*}-l\right)+l^{2}\right)>0$ (the eigenvalues $\lambda_{1,2}$ are real, and one of them is positive) for

$$
\frac{b_{*}}{l}<k_{-} \quad \text { or } \quad \frac{b_{*}}{l}>k_{+} .
$$

It is easy to check that for $\gamma \in(2,4)$ condition (12) is more restrictive than (13), whereas for $\gamma>4$ condition (13) is more restrictive. Therefore the proof is over.

One can readily check that if $b_{*} \in\left[\frac{1-\sqrt{2}}{2} l, \frac{1+\sqrt{2}}{2} l\right]$, then all eigenvalues have zero real part. Thus, for this range of parameters the linear analysis does not give an answer about stability of the equilibrium. Nevertheless, the following theorem holds. 
Theorem 2.2. [12] Equilibrium (9) is stable in the Lyapunov sense for any $\gamma>1$ if

$$
\frac{b_{*}}{l} \in(0,1) .
$$

The proof is based on the explicit construction of the Lyapunov function (see the details in [12]).

Very recently the following theorem was proved.

Theorem 2.3. [14] For $\gamma=2$ equilibrium (9) is stable in the Lyapunov sense for all

$$
b_{*} \in\left(\frac{1-\sqrt{2}}{2} l, \frac{1+\sqrt{2}}{2} l\right),
$$

and unstable otherwise.

The proof is quite technical and based on the use of the Lagrangian coordinates.

We do not dwell here on the study of stability of equilibria (10), (11), it is not trivial. The linear analysis implies that the matrix of linearization has a couple of eigenvalues for $\pm \frac{1}{c_{*}} \sqrt{c_{*} l\left(\left(a_{*}^{2}+c_{*}^{2}\right) \gamma-c_{*} l\right)}$ for (10) and $\pm \sqrt{-b_{*} l(\gamma+1)}$ for (11), whereas the other eigenvalues have the zero real part. Thus, one can easily find the range of parameters guaranteing instability. The proof of instability in the rest of cases is much more delicate.

2.2. $\delta=1, s=1$. To reduce computations and obtain analytical result, we dwell on a particular case $s=1$. We have the following real equilibria:

$$
\begin{gathered}
a=d=0, b=-c=b_{*}, A=C=\frac{\left(\omega-b_{*}\right)^{2}}{2 c_{0}}, B=0, \\
a=a_{*}, c=c_{*}, b=\frac{\omega^{2}-a_{*}^{2}}{c_{*}}, d=-a_{*}, A=\frac{c_{*} \omega}{c_{0}}, B=-\frac{2 a_{*} \omega}{c_{0}}, C=\frac{\omega\left(a_{*}^{2}-\omega^{2}\right)}{c_{*} c_{0}},
\end{gathered}
$$

for $c_{*}=0$ the later equilibrium should be completed by

$$
a= \pm \omega, b=b_{*}, d=\mp \omega, A=0, B=\mp \frac{2 \omega^{2}}{c_{0}}, C=-\frac{\omega b_{*}}{c_{0}} .
$$

Thus, (14) and (15), (16) correspond to (9) and (10), (11) for $\delta=0$.

One can easily see that the "anticyclonic high" domain of parameters disappears from (14). Thus, the situation is similar to the case $\delta=0$ with $l=0$ (the plane does not rotate).

Equilibria (15), (16) correspond to the saddle point of pressure.

Theorem 2.4. Equilibrium (14) is unstable for all values of parameter $b_{*}$.

Proof. The eigenvalues of matrix of linearization for the system (7), (8) at the point (14) are the following:

$$
\lambda_{1}=0, \lambda_{2,3}= \pm \sqrt{2(\gamma-2)}\left(\omega-b_{*}\right), \lambda_{4,5,6,7}= \pm \sqrt{-4 b_{*} \omega \pm 2\left(b_{*}^{2}-\omega^{2}\right) i}
$$


Therefore for $b_{*} \neq \omega$ there is an eigenvalue with a positive real part. The only possibility to have the zero real part is $b_{*}=\omega$. Let us prove that this case is also unstable. First of all we notice that if $b_{*}=\omega$, than $A_{*}=C_{*}=0$. If we fix $A=B=C=0$, we will get a subset of solutions of the full system (17), (8), corresponding to $R=0$, that is

$$
\dot{Q}+Q^{2}+2 \omega L Q-\omega^{2} \mathcal{E}=0,
$$

where $\mathcal{E}$ is the identity matrix. We are going to find a small perturbation of equilibrium of this matrix Riccati equation which results in a finite time blow up. For this raison we first make a change $Q=\tilde{Q}-\omega L$, since $Q=-\omega L$ is the steady state, corresponding to the equilibrium point. For $\tilde{Q}$ we get the matrix Bernoulli equation

$$
\dot{\tilde{Q}}=-\tilde{Q}^{2}+\omega \tilde{Q} L-\omega L \tilde{Q},
$$

where the equilibrium is shifted to the zero point. Equation (17) can be reduced to the following linear equation for $U=\tilde{Q}^{-1}$ (see, e.g [5]):

$$
\dot{U}=\omega(U L-L U)+E .
$$

The initial condition $U(0)$ can be derived from $\tilde{Q}(0)$ provided $\operatorname{det} \tilde{Q}(0) \neq 0$. Equation (18) can be explicitly solved. Let us choose initial perturbations of the equilibrium $\tilde{Q}=0$ as $\tilde{Q}_{11}=\tilde{Q}_{22}=0, \tilde{Q}_{12}=\tilde{Q}_{21}=\varepsilon$. The components of $U(t)$ are polynomials with respect to $t$ up to the second order (we skip the standard computations), nevertheless, the components $\tilde{Q}(t)=U^{-1}(t)$ contain $\operatorname{det} U(t)$ in the denominator. For our specific choice of perturbation $\operatorname{det} U(t)=\left(\frac{2 \omega}{\varepsilon}+1\right)^{2} t-\frac{1}{\varepsilon^{2}}$ and the components of $\tilde{Q}(t)$ tend to infinity as $t \rightarrow(2 \omega+\varepsilon)^{-2}$. Thus, the theorem is proved.

For equilibria (15) and (16) the spectra of matrices of linearization contain three zeros and the quadriple

$$
\pm \frac{1}{c_{*}} \sqrt{c_{*} w\left(\gamma z_{*} \pm \sqrt{z_{*}^{2} \gamma^{2}+32(\gamma-2) c_{*} w^{2}}\right)}, \quad z_{*}=a_{*}^{2}+c_{*}^{2}-w^{2}
$$

(for (15) ) and

$$
\pm \sqrt{\left.-b_{*} w \gamma \pm \omega \sqrt{b_{*}^{2} \gamma^{2}+32(\gamma-2) w^{2}}\right)}
$$

(for (16)). Thus, one can find conditions on the parameters ensuring instability of the equilibria. Let us notice that these conditions strongly depend on the heat ratio $\gamma$. For example, (16) is always unstable for $\gamma>2$. The stability for the linearly neutral cases is not studied, nevertheless, it is no reason to expect stability for the saddle points of the pressure. 
2.3. $\delta=1, s=\sin \theta$. In the general case the equilibria of system (17), (8) can be found as roots of algebraic equations of a higher order. We do not list them all, nevertheless let us mention the following nontrivial family of equilibria depending only on one parameter $c_{*}$ :

$$
\begin{array}{r}
a=0, b=z, c=c_{*}, d=0, \\
A=\frac{1}{2 c_{0}}\left(2 c_{*} w s-z c_{*}+w^{2}\right), B=0, C=\frac{1}{2 c_{0}}\left(w^{2} s^{2}-2 w s z-z c_{*}\right),
\end{array}
$$

where $z=z\left(c_{*}, w\right)$ is a root of the quadratic equation

$$
c_{*} z^{2}+\left(c_{*}^{2}-w^{2}\right) z-c_{*} s^{2} w^{2}=0 .
$$

This equation always has two real roots, therefore the properties of the equilibrium depend on the choice of the root. One equilibrium is a nonaxisymmetric vortex $\left(z=-c_{*}\right)$, another one is a saddle point $\left(z=\frac{w^{2}}{c_{*}}\right)$. If we set $s=1$, we get (14) and (15) for $a_{*}=0$.

For this case analytical computations are very cumbersome, nevertheless one can still find the eigenvalues of the matrix of linearization and see that the spectrum always contains a quadriple $\pm \alpha \pm \beta i$ with a nonzero $\alpha$, therefore, the equilibrium is unstable.

\section{Study of System (7), (8) In the LAgRAngian COORDinates}

In the present section we use the Lagrangian coordinates to show that for $\gamma=2$ and $s=1$ the solution can be found in terms of elliptic integrals.

Let $F=\left(F_{i k}\right)_{i, k=1 . .2}$ be the matrix of transfer from the Lagrangian coordinates $\mathbf{w}$ to the Eulerian ones $\mathbf{x}$, i.e. $\mathbf{x}(t, \mathbf{w})=F(t) \mathbf{w}$. This property is called the uniform deformation. Since $\mathbf{w}=F^{-1} \mathbf{x}$, then $\mathbf{u}=\dot{\mathbf{x}}=\dot{F} \mathbf{w}=$ $\dot{F} F^{-1} \mathbf{x}=Q \mathbf{x}$. Thus, the condition of uniform deformation is equivalent to condition (6).

System (77), (8) can be written in the Lagrangian coordinates as

$$
\ddot{F}_{i k}+\frac{\partial U}{\partial F_{i k}}+\sum_{j} \mathcal{L}_{i j} \dot{F}_{j k}-\delta \omega^{2} \mathcal{D} F_{i k}=0 .
$$

Here $U$ is the internal energy of gas, $U=U_{0}(|\operatorname{det} F|)^{-\gamma} \operatorname{det} F, U_{0}=$ const $>$ $0, F$ is nondegenerate, the derivatives are taken with respect to time.

For $l=\delta=0$ system (19) was considered extensively in the literature both in $3 \mathrm{D}$ and $2 \mathrm{D}$ cases. It models an expansion of a gas ellipsoid (or elliptic cylinder in $2 \mathrm{D}$ case) into vacuum. The problem originates from the paper of L.V.Ovsyannikov [8] (1956), the state of art and respective references can be found in [3]. In [4, [1] the first integrals of the system (19) were found for $l=\delta=0$. The integrals are the following:

$$
\begin{gathered}
\frac{1}{2} \sum_{i, k}\left(\dot{F}_{i k}\right)^{2}+U=E \\
F_{11} \dot{F}_{21}+F_{12} \dot{F}_{22}-F_{21} \dot{F}_{11}-F_{22} \dot{F}_{12}=J,
\end{gathered}
$$




$$
F_{11} \dot{F}_{12}+F_{21} \dot{F}_{22}-F_{12} \dot{F}_{11}-F_{22} \dot{F}_{21}=K \text {. }
$$

Moreover, for the case of one-atomic gas (for $x \in \mathbb{R}^{2}$ it corresponds to $\gamma=2$ ) in [1] it was shown the existence of a supplemental first integral

$$
G=\sum_{i, k} F_{i k}^{2}=2 E t^{2}+k_{1} t+k_{0}
$$

where $k_{0}$ and $k_{1}$ are constants. It gives a possibility to find exact solution to system (19) in the 2D case. In [2] an oscillating regime of (19) were established.

\subsection{First integrals.}

Theorem 3.1. System (19) has the integral of energy

$$
\tilde{E}=E-\frac{1}{2} \omega^{2}\left(F_{11}^{2}+F_{12}^{2}+k\left(F_{21}^{2}+F_{22}^{2}\right)\right) \text {. }
$$

For $s=1$ the system (19) has three first integrals:

$$
\tilde{E}=E-\frac{1}{2} \omega^{2} G, \quad A=J+\omega G, \quad B=K-2 \omega \operatorname{det} F .
$$

For $\gamma=2$, there exists a supplemental first integral

$$
G=\sum_{i, k} F_{i k}^{2}=2(\tilde{E}+\omega A) t^{2}+k_{1} t+k_{0},
$$

where $k_{0}$ and $k_{1}$ are constants.

Proof. To prove (21) we multiply every equation of system (19) by $\dot{F}_{i k}$ and add the results. We get

$$
\left(\ddot{F}_{i k} \dot{F}+\frac{\partial U}{\partial F}+L \dot{F}+\omega^{2} \mathcal{D} F\right) \dot{F}=\tilde{E}^{\prime}=0 .
$$

To prove the existence of integrals $A$ and $B$ from (22) with $k=1$ we check that

$$
-F_{11} \frac{\partial U}{\partial F_{21}}-F_{12} \frac{\partial U}{\partial F_{22}}+F_{21} \frac{\partial U}{\partial F_{11}}+F_{22} \frac{\partial U}{\partial F_{12}}=0 .
$$

Indeed, we denote $\eta=\operatorname{det} F$ and get

$$
\begin{gathered}
-F_{11} \frac{\partial U}{\partial F_{21}}-F_{12} \frac{\partial U}{\partial F_{22}}+F_{21} \frac{\partial U}{\partial F_{11}}+F_{22} \frac{\partial U}{\partial F_{12}}= \\
\left(F_{11} F_{12}-F_{12} F_{11}+F_{21} F_{22}-F_{22} F_{21}\right) U_{\eta}^{\prime}=0 .
\end{gathered}
$$

Then we differentiate $J$ and take into account (19) to obtain

$$
\begin{gathered}
\dot{J}=F_{11} \ddot{F}_{21}+F_{12} \ddot{F}_{22}-\ddot{F}_{11} F_{21}-\ddot{F}_{12} F_{22}= \\
F_{11}\left(-l \dot{F}_{11}-\frac{\partial U}{\partial F_{21}}-\omega^{2} F_{21}\right)+F_{12}\left(-l \dot{F}_{12}-\frac{\partial U}{\partial F_{22}}-\omega^{2} F_{22}\right)- \\
F_{21}\left(l \dot{F}_{21}-\frac{\partial U}{\partial F_{11}}-\omega^{2} F_{11}\right)-F_{22}\left(l \dot{F}_{22}-\frac{\partial U}{\partial F_{22}}-\omega^{2} F_{12}\right)=-l \sum_{i, j} F_{i j} \dot{F}_{i j}=-\frac{l}{2} \dot{G} .
\end{gathered}
$$

It implies $J+\frac{l}{2} G=$ const and (22) , if we take into account that $l=2 \omega$. 
The proof of existence of the integral $B$ is analogous, since

$$
-F_{11} \frac{\partial U}{\partial F_{12}}-F_{21} \frac{\partial U}{\partial F_{22}}+F_{12} \frac{\partial U}{\partial F_{11}}+F_{22} \frac{\partial U}{\partial F_{21}}=0 .
$$

To prove (23) we notice that for $\gamma=2$ by the Euler theorem $\sum_{i, j} F_{i j} \frac{\partial U}{\partial F_{i j}}=$ $2 U$, since $U=U_{0}(\operatorname{det} F)^{-1}$ is a homogeneous function of order -2 .

Thus,

$$
\begin{gathered}
\frac{\ddot{G}}{2}=\sum_{i, j}\left(\dot{F}_{i j}^{2}+F_{i j} \ddot{F}_{i j}\right)= \\
=\sum_{i, j} \dot{F}_{i j}^{2}+F_{11}\left(l \dot{F}_{21}-\frac{\partial U}{\partial F_{11}}+\omega^{2} F_{11}\right)+F_{12}\left(l \dot{F}_{22}-\frac{\partial U}{\partial F_{12}}+\omega^{2} F_{12}\right)+ \\
F_{21}\left(-l \dot{F}_{11}-\frac{\partial U}{\partial F_{21}}+\omega^{2} F_{21}\right)+F_{22}\left(-l \dot{F}_{12}-\frac{\partial U}{\partial F_{22}}+\omega^{2} F_{22}\right)= \\
(2 E-2 U)+l\left(F_{11} \dot{F}_{21}+F_{12} \dot{F}_{22}-F_{21} \dot{F}_{11}-F_{22} \dot{F}_{12}\right)-\sum_{i, j} F_{i j} \frac{\partial U}{\partial F_{i j}}+\omega^{2} G= \\
2 E+l J-w^{2} G=2 \tilde{E}+l J+2 \omega^{2} G=2(\tilde{E}+\omega A)=\text { const. }
\end{gathered}
$$

It implies (23).

Remark 3.1. We can see that (23) and (20) are similar, they are polynomials of order 2 with respect to $t$. For $\delta=0$ the situation is absolutely different. Namely, as is shown in [14],

$$
G=M \sin \left(l t+\phi_{0}\right)+\frac{4 \tilde{E}+2 l A}{l^{2}}
$$

with constant $M$ and $\phi_{0}$, it is periodic. One can see that the in the case $\delta=1, k=1$ the influence of rotation is neutralized.

\subsection{Representation of solution.}

Theorem 3.2. For $\delta=0$ and $k=1$ the system (19) can be reduced to one $O D E$

$$
u^{\prime}(t)= \pm \frac{1}{s^{2}(t)} \sqrt{f(u)}
$$

and integrated. Here $f(u)=D-\frac{4 U_{0}}{\sin 2 u}-\frac{A^{2}+B^{2}+2 A B \sin 2 u}{\cos ^{2} 2 u}, D=\frac{4(\tilde{E}+\omega A) k_{0}-k_{1}^{2}}{4}=$ const, $s^{2}(t)=G(t)$.

Proof. The change of variables

$$
\left(\begin{array}{ll}
F_{11} & F_{12} \\
F_{21} & F_{22}
\end{array}\right)=\left(\begin{array}{cc}
\cos v & -\sin v \\
\sin v & \cos v
\end{array}\right)\left(\begin{array}{cc}
s \cos u & 0 \\
0 & s \sin u
\end{array}\right)\left(\begin{array}{cc}
\cos w & -\sin w \\
\sin w & \cos w
\end{array}\right)
$$

reduces the system of first integrals (22) to

$$
\begin{gathered}
\frac{s^{2}}{2}\left(\left(u^{\prime}\right)^{2}+\omega^{2}+\left(v^{\prime}\right)^{2}+2 \sin (2 u) v^{\prime} w^{\prime}+\left(w^{\prime}\right)^{2}\right)+\frac{\left(s^{\prime}\right)^{2}}{2}+\frac{2 U_{0}}{s^{2} \sin 2 u}=\tilde{E}, \\
\left(v^{\prime}+\frac{l}{2}\right)+\sin (2 u) w^{\prime}=\frac{A}{s^{2}},
\end{gathered}
$$


Further, $G=\sum_{i, k} F_{i k}^{2}=s^{2}(t)$, therefore we can use the expression (23).

From (27) we obtain

$$
\begin{aligned}
& v^{\prime}=\frac{1}{s^{2}} \cdot \frac{A+B \sin 2 u}{\cos ^{2} 2 u}-\frac{l}{2}, \\
& w^{\prime}=-\frac{1}{s^{2}} \cdot \frac{B+A \sin 2 u}{\cos ^{2} 2 u} .
\end{aligned}
$$

Together with (26) it implies (25).

Remark 3.2. The solution $u(t)$ of (25) contains elliptic integrals. Indeed, if we denote $r=\sin 2 u$, then $\dot{u}=\frac{\dot{r}}{2 \sqrt{1-r^{2}}}$ and $\cos ^{2} 2 u=1-r^{2}$. Thus, (25) can be reduced to $\frac{\dot{r}}{2}= \pm \frac{1}{s^{2}} \sqrt{\frac{f(r)}{r}}$, where $f(r)$ is a polynomial of the third order. Thus,

$$
\begin{gathered}
\int_{0}^{t} \frac{1}{s^{2}(\tau)} d \tau= \\
=\mp \frac{r_{1}}{\sqrt{-D r_{2}\left(r_{3}-r_{1}\right)}}\left(F\left(\sqrt{\frac{r\left(r_{3}-r_{1}\right)}{r_{3}\left(r-r_{1}\right)}}, \sqrt{\frac{r_{3}\left(r_{1}-r_{2}\right)}{r_{2}\left(r_{1}-r_{3}\right)}}\right)-\Pi\left(\frac{r_{3}}{r_{1}-r_{3}}, \sqrt{\frac{r\left(r_{3}-r_{1}\right)}{r_{3}\left(r-r_{1}\right)}}, \sqrt{\frac{r_{3}\left(r_{1}-r_{2}\right)}{r_{2}\left(r_{1}-r_{3}\right)}}\right)\right),
\end{gathered}
$$

where $r_{1}, r_{2}, r_{3}$ are the roots of equation $f(r)=0, F$ and $\Pi$ are normal elliptic Legendre integrals of the first and third order in the Jacobi form. The roots are simple, otherwise among them there is one that corresponds to equilibrium and the solution does not exist in its neighborhood.

Remark 3.3. It is easy to check that if we set $s^{2}=G=2 E t^{2}+k_{1} t+k_{0}$ and $l=\omega=0$, then $D=\frac{8 E k_{0}-k_{1}^{2}}{4}$. Thus, we get the result of of [1], where the left-hand side of the formula is $\int \frac{d t}{2 E t^{2}+k_{1} t+k_{0}}=\frac{1}{2 E \beta} \arctan \frac{4 E t+k_{1}}{\beta}$, with $\beta^{2}=4 D>0$.

Remark 3.4. If we know the solution to (25), we can go back to the Eulerian coordinates and find $\mathbf{u}$. Indeed, the functions $v$ and $w$ can be found from (27). Thus, the coefficients of the matrix $F$ are known, $Q=\dot{F} F^{-1}$ and $\mathbf{u}=Q \mathbf{x}$.

Remark 3.5. If $u$ tends to zero, then $\operatorname{det} F$ tends to zero and the components of $Q$ tends to infinity. Since these components have sense of derivatives of the solution, then it implies a blow-up. Thus, for the model of vortex with uniform deformation the instability of an equilibrium in fact implies blow-up of derivatives. One can hypothesize that the same phenomenon holds for a localized vortex in a gas, i.e. the instability leads to formation of singularities (see [13]). 


\section{Discussion}

We prove that if we take into account a small correction due to centrifugal force usually neglected in the $l$-plane model of atmosphere, we drastically change properties of a specific class of vortices. However, this does not mean that the generally accepted model is erroneous. It is well known that it is approximate and adequately describes only processes of relatively small scale. The model we are considering is also approximate, and an accurate description of the processes of the vortex dynamics of the scale of tropical cyclones can be carried out only within the framework of equations on a sphere.

In addition, vortex solutions with a uniform deformation, on which the difference in stability is manifested, have infinite energy. That is, they are nonphysical on the whole plane. To notice the effect described in this paper, it is necessary that the members $\mathcal{L} \mathbf{u}$ and $\omega^{2} \mathbf{x}$ ) (see (5)) have the same order. For this, the linear velocity profile must be maintained inside the vortex within a radius of the order of $\frac{l}{\omega^{2}} \mathbf{u} \approx 100 \mathrm{~km}$ (the typical value of velocity $\mathbf{u}$ is about $10 \mathrm{~m} / \mathrm{sec}$ ). This is not observed in nature, in fact, this radius is about $30 \mathrm{~km}$. Near the equator, where the Coriolis parameter is small, stable atmospheric vortices are not observed. Perhaps the effect described in this paper may be one of the factors of their destabilization.

Solutions with a linear velocity profile may seem only an interesting mathematical object. However, it is not quite like that. In fact, solutions of this class have enormous applications in astrophysics (e.g. [2]), in the point blast theory [6], etc. For us, their application to geophysics is important. The motivation is the fact that, near the center of a large atmospheric vortex in its conservative stage, the structure of wind velocity is linear. There are attempts to use this fact to predict the motion of the atmospheric vortex [9], [10]. Note that the full structure of stationary vortices is very diverse [1].

\section{ACKNOWLEGMENTS}

The first author thanks Nikolai Leontiev for a stimulating discussion.

\section{REFERENCES}

[1] Anisimov, S.I., Lysikov, Iu.I.: Expansion of a gas cloud in vacuum. J. Appl. Math. Mech. 34(5), 882-885 (1970).

[2] Bogoyavlensky, O.I.: Methods in the qualitative theory of dynamical systems in astrophysics and gas dynamics. Springer Series in Soviet Mathematics (1985).

[3] Borisov, A. V., Mamaev, I. S., Kilin, A. A.: The Hamiltonian dynamics of self-gravitating liquid and gas ellipsoids, Regul. Chaot. Dyn. 14, 179-217 (2009) doi.org/10.1134/S1560354709020014

[4] Dyson, J.F.: Dynamics of a spinning gas cloud. J. Math. Mech. 18, 91-101(1968).

[5] Egorov, A.I.: Riccati Equations. Pensoft Publishers (2007)

[6] Korobeinikov, V.P.: Problems of point blast theory. American Institute of Physics (1991) 
[7] Obukhov, A.M.: On the geostrophical wind. Izv.Acad.Nauk (Izvestiya of Academie of Science of URSS), Ser. Geography and Geophysics, XIII, 281-306 (1949).

[8] Ovsyannikov, L.V.: New solution of hydrodynamics equations, Dokl. Akad. SSSR 111, 47-49(1956).

[9] Rozanova, O.S., Yu, J.-L., Hu, C.-K.: Typhoon eye trajectory based on a mathematical model: Comparing with observational data. Nonlinear Analysis: Real World Applications. 11, 1847-1861 (2010).

[10] Rozanova, O.S., Yu, J.-L., Hu, C.-K.: On the position of vortex in two-dimensional model of atmosphere. Nonlinear Analysis: Real World Applications, 13, 1941-1954 (2012)

[11] Rozanova, O.S.: Frozen and almost frozen structures in the compressible rotating fluid. Bulletin of the Brazilian Mathematical Society, New Series. 47, 715-726 (2015)

[12] Rozanova, O.S., Turzynsky, M.K.: On Systems of Nonlinear ODE Arising in Gas Dynamics: Application to Vortical Motion. In: Differential and Difference Equations with Applications. Springer Proceedings in Mathematics and Statistics, 230, 387-398 (2018). doi.org/10.1007/978-3-319-75647-9_32

[13] Rozanova, O.S., Turzynsky, M.K.: Nonlinear stability of localized and non-localized vortices in rotating compressible media. In: Theory, Numerics and Applications of Hyperbolic Problems. Springer Proceedings in Mathematics and Statistics. 236, 567580 (2018). doi.org/10.1007/978-3-319-91548-7_41

[14] Turzynsky, M.K.: On properties of solutions with uniform deformation of the system of gas dynamics equations on a rotating plane, submitted.

[15] Vallis, G.K.: Atmospheric and oceanic fluid dynamics. Fundamentals and large-scale circulation. Cambridge University Press (2006).

Mathematics and Mechanics Faculty, Moscow State University, Moscow 119991, Russia

E-mail address: rozanova@mech.math.msu.su 